\title{
GAMMA-RAY BURST ENERGETICS AND THE GAMMA-RAY BURST HUBBLE DIAGRAM: PROMISES AND LIMITATIONS
}

\author{
J. S. Bloom, ${ }^{1,2}$ D. A. Frail, ${ }^{3}$ and S. R. Kulkarni ${ }^{4}$ \\ Received 2003 January 9; accepted 2003 May 20
}

\begin{abstract}
We present a complete sample of 29 gamma-ray bursts (GRBs) for which it has been possible to determine temporal breaks (or limits) from their afterglow light curves. We interpret these breaks within the framework of the uniform conical jet model, incorporating realistic estimates of the ambient density and propagating error estimates on the measured quantities. In agreement with our previous analysis of a smaller sample, the derived jet opening angles of those 16 bursts with redshifts result in a narrow clustering of geometrically corrected gamma-ray energies about $\mathscr{E}_{\gamma}=1.33 \times 10^{51} \mathrm{ergs}$; the burst-to-burst variance about this value is 0.35 dex, a factor of 2.2. Despite this rather small scatter, we demonstrate in a series of GRB Hubble diagrams that the current sample cannot place meaningful constraints upon the fundamental parameters of the universe. Indeed, for GRBs to ever be useful in cosmographic measurements, we argue the necessity of two directions. First, GRB Hubble diagrams should be based upon fundamental physical quantities such as energy, rather than empirically derived and physically ill-understood distance indicators (such as those based upon prompt burst time-profiles and spectra). Second, a more homogeneous set should be constructed by culling subclasses from the larger sample. These subclasses, although now first recognizable by deviant energies, ultimately must be identifiable by properties other than those directly related to energy. We identify a new subclass of GRBs ("f-GRBs") that appear both underluminous by factors of at least 10 and exhibit a rapid fading $\left(f_{\nu} \propto t^{-2}\right)$ at early times ( $t \lesssim 0.5$ day). About $10 \%-20 \%$ of observed long-duration bursts appear to be f-GRBs.
\end{abstract}

Subject headings: cosmological parameters — cosmology: miscellaneous — cosmology: observations distance scale — gamma rays: bursts — supernovae: individual (SN 1998bw)

On-line material: color figures

\section{INTRODUCTION}

The observational establishment of the extra-Galactic origin of gamma-ray bursts (GRBs; Metzger et al. 1997) enlivened the possibility of exploiting GRBs for cosmological investigations. The discovery of GRBs at high redshifts (GRB 971214, $z=3.42$, Kulkarni et al. 1998a; GRB 000131, $z=4.51$, Andersen et al. 2000) further demonstrated that GRBs, like quasars, could be used to study the early universe and in particular the intergalactic medium, the interstellar medium of young galaxies, and star formation in dusty regions (see Loeb \& Barkana 2001 for a review).

The developments in the GRB field thus appear to mirror the early years of quasar astronomy. In both cases, astronomers were astonished by the unexpectedly large brilliance. In both cases, the fields split into two camps: many who used the objects for cosmology and some who were intrigued by the workings of the central engine.

However, the large luminosity function of quasars precluded their use as a means to determine the geometry of the universe, i.e., for cosmography. The bewildering diversity of GRBs in almost any respect-fluence, peak luminositylikewise seemed to make them unsuitable for this purpose.

\footnotetext{
${ }^{1}$ Harvard Society of Fellows, 78 Mount Auburn Street, Cambridge, MA 02138 .

${ }^{2}$ Harvard-Smithsonian Center for Astrophysics, MC 20, 60 Garden Street, Cambridge, MA 02138

${ }^{3}$ National Radio Astronomy Observatory, Socorro, NM 87801.

${ }^{4}$ Palomar Observatory 105-24, California Institute of Technology, Pasadena, CA 91125.
}

Frail et al. (2001, hereafter FKS01) presented a complete sample of GRBs with good afterglow follow-up and known redshifts. Most afterglows exhibit a steepening, seen either at optical or radio wavelengths, which is expected if the explosion geometry is conical (opening half-angle, $\theta_{\text {jet }}$ ) rather than spherical (Rhoads 1997a; Sari, Piran, \& Halpern 1999). FKS01 interpret the breaks making several simplifying assumptions (constant ambient density, constant gamma-ray conversion efficiency) and find the surprising result that the seemingly most energetic bursts, $E_{\text {iso }}(\gamma)$ (isotropic-equivalent gamma-ray energy release), possess the smallest beaming factor, $f_{b}=1-\cos \left(\theta_{\text {jet }}\right)$. In that sample, the true energy release, $E_{\gamma}=f_{b} E_{\text {iso }}(\gamma)$, was strongly clustered.

This finding - that GRBs are standard-energy explosions-opens up the possibility of using GRBs and their afterglows for cosmography. Indeed, in the 2001 August meeting at Garching ${ }^{5}$ we noted that a Hubble diagram constructed from $E_{\gamma}$ in the FKS01 sample had a smaller scatter $(0.31 \mathrm{dex}=0.79 \mathrm{mag})$ compared to the dust-corrected $B$ band peak magnitude of Type Ia supernovae (SNe Ia) as of 1992 ( $\sigma=0.84$ mag; see Fig. 3 of van den Bergh \& Pazder 1992). Recently, Schaefer (2003) presented another GRB Hubble diagram based on bursts that have two empirical distance indicators: the variability index (Fenimore \& Ramirez-Ruiz 2000; Norris, Marani, \& Bonnell 2000; Reichart et al. 2001) and the lag-luminosity correlation (Norris 2002). The Schaefer analysis find a similarly small

\footnotetext{
${ }^{5}$ See http://www.mpa-garching.mpg.de/ light/
} 
scatter about a standard candle. The current distanceindicator sample is small (nine sources) and restricted to GRBs with known redshifts and gamma-ray observations from $B A T S E$.

As the experience with previous efforts at cosmography has shown, standard candles whose physics is understood (e.g., Cepheid variables) have proved more useful and robust than those that were based on poorly understood or unphysical phenomena (e.g., brightest stars, number counts). For SNe Ia the physics of the explosion is reasonably well understood. Even so, for SNe Ia, it is still important to understand the intrinsic diversity (i.e., the distinction between the normal-energy $\mathrm{SNe}$ and the so-called Branch peculiars; Branch, Fisher, \& Nugent 1993), to eliminate the large fraction ( $36 \%$; Li et al. 2001) of outliers on a sound empirical basis, and thereby increase the precision of the standard candle. Ultimately, the distance indicator must be calibrated to high enough accuracy to be competitive with precision cosmological experiments such as WMAP (Spergel et al. 2003).

As with SNe Ia, the apparent constancy of the prompt energy release in GRBs is plausible on physical grounds. Furthermore, indications of a constant energy release come from studies of GRB afterglows (Piran et al. 2001; Panaitescu \& Kumar 2002; Berger, Kulkarni, \& Frail 2003), a physically distinct emission process from the prompt burst emission. It is reasonable to expect that complementary afterglow data (as with photometry and spectroscopy of $\mathrm{SNe}$ Ia) would allow us to identify potential families, or subclasses, in GRBs and thus compile more homogeneous samples.

With this motivation and noting that the sample of GRBs with quality afterglow data has increased since the FKS01 analysis (from 17 to 29), we revisit the current state of the GRB $E_{\gamma}$ Hubble diagram paying careful attention to the error analysis and identifying potential subclasses of GRBs.

\section{PHYSICAL INTERPRETATIONS FOR OBSERVED TEMPORAL BREAKS}

Temporal breaks (secular steepening of a light curve) have been observed or inferred at optical, X-ray, and radio wavelengths in a number of burst afterglows. When wellsampled broadband imaging exists, these breaks appear to be achromatic or very nearly achromatic. The (near) achromaticity is suggestive of a geometric rather than an emissivity effect (such as expected from a hydrodynamically evolving synchrotron shock).

At present, two different geometric pictures are used to interpret the existence of $t_{\text {break }}$, the characteristic break time between two asymptotic power-law decays (see Kumar \& Granot 2003 for a comprehensive review). In one interpretation (the "top-hat" model), the energy per unit solid angle is constant within some cone with a half-angle opening $\theta_{\text {jet }}$ and falls to zero outside this angle. The break occurs at a time when the bulk Lorentz factor of the shock has slowed to $\Gamma \approx 1 / \theta_{\text {jet }}$ (Rhoads 1997b; Sari et al. 1999). In the other interpretation (the "universal jet" or "structured jet" model), the energy per unit solid angle varies across the face of the jet and the observer lies at an angle $\Theta_{\text {obs }}$ from the jet axis (Mészáros, Rees, \& Wijers 1998; Zhang \& Mészáros 2002). Here the break occurs at a time when the bulk Lorentz factor of the shock in the direction of the observer slows to $\Gamma\left(\Theta_{\text {obs }}\right) \approx 1 / \Theta_{\text {obs }}$. The present data cannot yet distinguish between the top-hat and universal jet models (Rossi, Lazzati, \& Rees 2002). The observational implications of a hybrid of the two models have only recently been explored (Kumar \& Granot 2003).

The inference of $\theta_{\text {jet }}$ or $\Theta_{\text {obs }}$ from $t_{\text {break }}$ requires an estimate of the evolution of $\Gamma$ as a function of observer time. The dynamical evolution of $\Gamma$ is theoretically prescribed by the relativistic Sedov solution (Blandford \& McKee 1976). This solution is dependent upon the isotropic-equivalent energy in the shock and the nature of the ambient medium into which the shock propagates for $t<t_{\text {break }}$ (e.g., Sari, Piran, \& Narayan 1998). The isotropic-equivalent energy in the prompt burst is measured using the observed fluence, prompt-burst spectrum, and distance to the source. The nature of the ambient medium is generally assumed to be homogeneous - density as a function of distance $r$ from the explosion site as $\rho(r)=n_{0} r^{0}$-or wind-stratified $[\rho(r)=$ $\left.A^{*} r^{-2}\right]$. Light curves of the more general case $\rho(r) \propto r^{s}$ have also been explored (e.g., Panaitescu, Mészáros, \& Rees 1998).

Of the $\sim 30$ well-studied GRB afterglow to date, there have been only $\approx 2$ bursts for which a wind-stratified media are strongly favored statistically over homogeneous media (GRB 980519: Frail et al. 2000b; GRB 011121: Price et al. 2002b). In the remainder, winds are either excluded (e.g., 990510: Harrison et al. 1999, Panaitescu \& Kumar 2002; 990123: Chevalier \& Li 2000) or allowed at similar significances as homogeneous profiles (e.g., 980329; Yost et al. 2002; see also Panaitescu \& Kumar 2002). Recently, Panaitescu \& Kumar (2003) have reanalyzed the afterglow of GRB 990510 and showed an improved consistency with the wind hypothesis by taking into account a structured jet.

In what follows, we construct a set of geometry-corrected energies for GRBs, following from the formalism of FKS01, under the assumption that the early afterglow propagates into a density of $\rho(r)=n_{0} r^{0}$ (we later address the differences when, instead, a wind-stratified medium is assumed). We also assume a top-hat model for the structure of the jet but note the resulting energy determinations are identical under the universal jet model interpretation when the energy per unit solid angle drops as $\Theta^{-2}$ (Zhang \& Mészáros 2002; see also Kumar \& Granot 2003 and references therein).

\section{FORMALISM AND ERROR ANALYSIS}

Following the definitions and notation of FKS01 and Bloom, Frail, \& Sari (2001b), the value for $E_{\gamma}$, the total prompt energy release in a certain bandpass, may be found from

$$
E_{\gamma}=S_{\gamma} \frac{4 \pi D_{l}^{2}}{(1+z)} k f_{b},
$$

where $S_{\gamma}$ is the fluence received in some observed bandpass and $D_{l}$ is the luminosity distance at redshift $z$. The quantity $k$ is a multiplicative correction of order unity relating the observed bandpass to the standard rest-frame bandpass, and the opening angle $\theta_{\text {jet }}$ is a function of the jet break time $t_{\text {break }} \equiv t_{\text {jet }}, E_{\text {iso }}(\gamma)$, and the ambient number density $n$ (see eq. [1] of FKS01). Since $\theta_{\text {jet }}$ is implicitly a function of $D_{l}, z, k$, and $S_{\gamma}$, the value for $E_{\gamma}$ is a complex function of observables.

Expressing all physical quantities in cgs units and making the analogy with astronomical magnitudes [distance 
modulus $\left.\equiv 5 \log _{10}\left(D_{l} / 10 \mathrm{pc}\right)\right]$, we rearrange equation (1) to give

$$
\begin{aligned}
\mathrm{DM}= & -2.5 \log _{10}\left[S_{\gamma} \frac{k}{1+z}\left(1-\cos \theta_{\mathrm{jet}}\right) 4 \pi\right] \\
& +2.5 \log _{10} E_{\gamma}-5 \log _{10} \frac{\mathrm{cm}}{10 \mathrm{pc}},
\end{aligned}
$$

where the DM is the "apparent GRB distance modulus." Assuming that the total energy output is constant $\left(\mathscr{E}_{\gamma}\right)$ from burst to burst, equation (2) becomes

$$
\mathrm{DM}=-2.5 \log _{10}\left[S_{\gamma} \frac{k}{1+z}\left(1-\cos \theta_{\text {jet }}\right) 4 \pi\right]+\mathrm{zp},
$$

with the zero point zp $=30.4940+2.5 \log _{10}\left(\mathscr{E}_{\gamma} / 1.5\right.$ foe $)$. The unit "foe" equals $10^{51}$ ergs.

The variance of $E_{\gamma}$ is a direct measure of the accuracy with which $E_{\gamma}$ can be used for cosmographic purposes. The most direct method to estimate the variance on $E_{\gamma}$ is to undertake a simultaneous analysis of the afterglow data and prompt burst emission to determine $f_{b}$ and $E_{\gamma}$. With this direct approach we do not have to worry about hidden correlations between different quantities.

Another approach is to carry out a simple error propagation of equation (1). The approach is straightforward but does require the assumption that there is no covariance in the measurement of $S_{\gamma}$ and the inference of $\theta_{\text {jet }}$. We justify this assumption by noting that $f_{b}$ is obtained from afterglow measurements, whereas $S_{\gamma}$ derives from the prompt emission - two very different phenomena. There could well be global correlations between the prompt burst emission and afterglow emission (e.g., the brightest bursts may be associated with bright afterglow), but we see no reason why our inference of $f_{b}$ should be observationally correlated to $S_{\gamma}$, beyond the small mathematical dependence of $f_{b}$ upon $E_{\gamma}$; see eq. [1] of Frail et al. 2001). Adopting this approach toward variance estimation, we find the fractional error on the $E_{\gamma}$ measurement from

$$
\begin{aligned}
\left(\frac{\sigma_{E_{\gamma}}}{E_{\gamma}}\right)^{2}= & \left(C_{\theta_{\text {jet }}}+1\right)\left[\left(\frac{\sigma_{S_{\gamma}}}{S_{\gamma}}\right)^{2}+\left(\frac{\sigma_{k}}{k}\right)^{2}\right] \\
& +C_{\theta_{\text {jet }}}\left[\left(\frac{\sigma_{n}}{n}\right)^{2}+\left(\frac{3 \sigma_{\text {jet }}}{t_{\text {jet }}}\right)^{2}\right],
\end{aligned}
$$

where

$$
C_{\theta_{\mathrm{jet}}}=\left[\frac{\theta_{\mathrm{jet}} \sin \theta_{\mathrm{jet}}}{8\left(1-\cos \theta_{\mathrm{jet}}\right)}\right]^{2} .
$$

The error in the $k$-correction $\left(\sigma_{k}\right)$ is given by equation (8) of Bloom et al. (2001b), and the error on the fluence $\left(\sigma_{S_{\gamma}}\right)$ is estimated from the prompt GRB observations. The errors on density $\left(\sigma_{n}\right)$ and the jet-break time $\left(\sigma_{t_{\text {iet }}}\right)$ are found from afterglow modeling. Again, we have assumed that the measurements of the four observables are uncorrelated. ${ }^{6}$ The error on the apparent GRB DM is $2.5 \times \sigma_{E_{\gamma}} / E_{\gamma}$. If

\footnotetext{
${ }^{6}$ The determinations of $t_{\text {jet }}$ and $n$ are inferred from afterglow modeling and thus are correlated to some degree. However, from the best observed bursts often $t_{\text {jet }}$ is found directly from optical/IR data, whereas density is best constrained with radio measurements. Thus, we expect the correlation on $t_{\text {jet }}$ and $n$ to be weak. Bloom et al. (2001b) discuss the possible correlations between $S_{\gamma}$ and $k$.
}

$\theta_{\text {jet }} \ll 1$, then $C_{\theta_{\text {jet }}} \approx 1 / 16$, implying that the fractional error on $E_{\gamma}$ can be easily dominated by uncertainties in the $k$-correction and $S_{\gamma}$.

\section{RESULTS}

In FKS01 we adopted a value of $n=0.1 \mathrm{~cm}^{-3}$ for the circumburst density for all bursts. One clear step toward improving the $E_{\gamma}$ measurements is a more realistic estimate of $n$ from broadband modeling of the afterglow light curves. We have now included the best density determinations that exist for about one-third of the GRBs in Table 1. In the past, large systematic differences in $n$ have been derived owing to incomplete data sets and the use of approximate relations for estimating afterglow parameters (Granot \& Sari 2002; Frail et al. 2003). However, with more precise photometric data and the increasing sophistication of afterglow modeling, the true diversity of number densities is becoming a well-established observation. Modeling yields estimates in the range $0.1 \mathrm{~cm}^{-3} \lesssim n \lesssim 30 \mathrm{~cm}^{-3}$, and there is now little support for extremes of either high or low density. Herein we make use of published estimates of $n$ and adopt a (new) canonical value of $10 \mathrm{~cm}^{-3}$ for all remaining events.

\subsection{Energetics and a Standard Energy}

In Table 2, we show the computed energetics values for $E_{\gamma}$ and the associated apparent GRB distance moduli, DM, for a cosmology with $\Omega_{\Lambda}=0.7, \Omega_{m}=0.3$, and $H_{0}=65$ $\mathrm{km} \mathrm{s}^{-1} \mathrm{Mpc}^{-1}$. Of the nine GRBs with measured $z, t_{\text {jet }}$, and $n$, the logarithmically weighted mean total energy release is 1.16 foe \pm 0.07 dex with a median energy of 1.33 foe. We therefore adopt a new standard energy of $\mathscr{E}_{\gamma}=1.33$ foe with an error of $\pm 0.07 \mathrm{dex}$. This standard value is dependent upon the choice of cosmology.

If we expand the sample to include all bursts with knowns redshifts and $t_{\text {jet }}$ but not density, the logarithmically weighted mean energy is 1.21 foe $\pm 0.08 \mathrm{dex}$. That these two mean values agree suggests that the assignment of $n=10$ $\mathrm{cm}^{-3}$ to the seven bursts with only $t_{\text {jet }}$ measurements reasonably reflects the density of the population without $n$ measurements. Figure 1 shows a histogram of $E_{\gamma}$ measurements, tightly clustered about $\mathscr{E}_{\gamma}$. Note that the adopted value for $\mathscr{E}_{\gamma}$, now found using a proper inclusion of density into the $E_{\gamma}$ formulation, is a factor of 2.7 higher than that found in FKS01. This is close to the analytic difference expected $\left(\approx 3.2\right.$ for $\left.\theta_{\text {ete }} \ll 1\right)$ by assuming $n=10 \mathrm{~cm}^{-3}$ instead of $n=0.1 \mathrm{~cm}^{-3}$, as in FKS01.

As seen in Figure 1, 11 of the 16 GRBs with $E_{\gamma}$ measurements fall within $5 \sigma$ of $\mathscr{E} \gamma$, and five bursts $(970508,990123$, 990510, 000418, and 011211) are outside $5 \sigma$ of $\mathscr{E} \gamma$. The variance of all 16 GRBs with measured $E_{\gamma}$ about our adopted standard energy is 0.35 dex and is dominated by the five outliers. Although there is no a priori reason to exclude these outlier bursts, the observed scatter of the more restricted sample without the outliers about $\mathscr{E}_{\gamma}$ is $0.13 \mathrm{dex}$, about a factor of $35 \%$.

\section{2. $\mathscr{E}_{\gamma}$ in a Cosmological Context}

The small apparent scatter of measured $E_{\gamma}$ values is continued evidence for a standard energy release in prompt gamma-ray emission, first noted in FKS01. As we pointed out in Garching 2001, this observation suggests that GRBs may be used as calibrated standard candles, useful, in 
TABLE 1

ObServed and Modeled Data for GRB Energy Determination

\begin{tabular}{|c|c|c|c|c|c|c|c|}
\hline \multirow[b]{2}{*}{ GRB } & \multirow[b]{2}{*}{$z$} & \multicolumn{2}{|c|}{ FLUENCE } & \multirow[b]{2}{*}{$k^{\mathrm{b}}$} & \multirow[b]{2}{*}{$\begin{array}{c}t_{\text {jet }} \\
\text { (day) }\end{array}$} & \multirow[b]{2}{*}{$\begin{array}{c}n \\
\left(\mathrm{~cm}^{-3}\right)\end{array}$} & \multirow[b]{2}{*}{ Reference } \\
\hline & & $\begin{array}{c}S_{\gamma} \\
\left(\times 10^{-6} \mathrm{ergs} \mathrm{cm}^{-2}\right)\end{array}$ & $\begin{array}{l}\text { Bandpass }^{\mathrm{a}} \\
\quad(\mathrm{keV})\end{array}$ & & & & \\
\hline 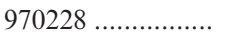 & 0.6950 & $11.70 \pm 2.00$ & $1.5,700$ & $0.830 \pm 0.041$ & & $\ldots$ & 1,2 \\
\hline 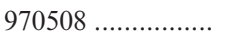 & 0.8349 & 3.17 & 20,2000 & $0.814 \pm 0.041$ & $25.00 \pm 5.00$ & 1.00 & $3,4,5,5$ \\
\hline 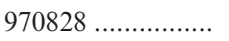 & 0.9578 & 96.00 & 20,2000 & $0.823 \pm 0.036$ & $2.20 \pm 0.40$ & $\ldots$ & $6,4,6$ \\
\hline $971214 \ldots \ldots \ldots \ldots \ldots$ & 3.4180 & 9.44 & 20,2000 & $0.804 \pm 0.057$ & $>2.5$ & $\ldots$ & $7,4,7$ \\
\hline $980326 \ldots \ldots \ldots \ldots \ldots . .$. & $\ldots$ & 0.92 & 20,2000 & $\ldots$ & $<0.4$ & $\ldots$ & 4,8 \\
\hline 980329 ................. & $\ldots$ & 55.10 & 20,2000 & $\ldots$ & $<1.0$ & $20.00 \pm 10.00$ & $4,9,9$ \\
\hline 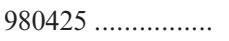 & 0.0085 & 3.87 & 20,2000 & $1.002 \pm 0.000$ & $\ldots$ & $\ldots$ & 10,4 \\
\hline 980519 ................... & $\ldots$ & 10.30 & 20,2000 & $\ldots$ & $0.55 \pm 0.17$ & $0.14_{-0.03}^{+0.32}$ & $4,11,12$ \\
\hline 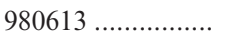 & 1.0969 & $1.71 \pm 0.25$ & 20,2000 & $0.863 \pm 0.110$ & $>3.1$ & $\ldots$ & $13,14,15$ \\
\hline $980703 \ldots \ldots \ldots \ldots \ldots . . .$. & 0.9662 & 22.60 & 20,2000 & $0.940 \pm 0.041$ & $3.40 \pm 0.50$ & $28.00 \pm 10.00$ & $16,4,17,17$ \\
\hline 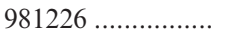 & $\ldots$ & $0.40 \pm 0.10$ & 40,700 & $\ldots$ & $>5.0$ & $\ldots$ & 18,19 \\
\hline 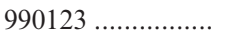 & 1.6004 & 268.00 & 20,2000 & $0.720 \pm 0.052$ & $2.04 \pm 0.46$ & $\ldots$ & $20,4,20$ \\
\hline 990506 .................. & 1.3066 & 194.00 & 20,2000 & $0.873 \pm 0.054$ & $\ldots$ & $\ldots$ & 21,4 \\
\hline 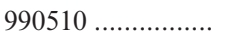 & 1.6187 & 22.60 & 20,2000 & $1.026 \pm 0.055$ & $1.20 \pm 0.08$ & $0.29_{-0.15}^{+0.11}$ & $22,4,23,12$ \\
\hline 990705 .................... & 0.8424 & $93.00 \pm 2.00$ & 40,700 & $1.279 \pm 0.098$ & $1.00 \pm 0.20$ & $\ldots$ & $24,25,26$ \\
\hline 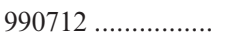 & 0.4331 & $6.50 \pm 0.30$ & 40,700 & $1.387 \pm 0.132$ & $>47.7$ & & $22,27,28$ \\
\hline 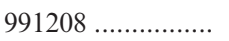 & 0.7055 & 100.00 & 25,10000 & $0.746 \pm 0.206$ & $<2.1$ & $18.00_{-6.00}^{+18.00}$ & $29,30,31,12$ \\
\hline $991216 \ldots \ldots \ldots \ldots \ldots \ldots$ & 1.0200 & 194.00 & 20,2000 & $0.877 \pm 0.042$ & $1.20 \pm 0.40$ & $4.70_{-1.80}^{+6.80}$ & $32,4,33,12$ \\
\hline $000131 \ldots \ldots \ldots \ldots \ldots$ & 4.5110 & 41.80 & 20,2000 & $0.646 \pm 0.074$ & $<3.5$ & $\ldots$ & 34,4 \\
\hline 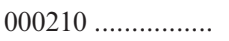 & 0.8463 & $61.00 \pm 2.00$ & 40,700 & $1.278 \pm 0.097$ & $>1.7$ & $\ldots$ & $35,35,34$ \\
\hline $000301 \mathrm{C} \ldots \ldots \ldots \ldots \ldots$ & 2.0335 & 4.10 & 25,1000 & $0.928 \pm 0.094$ & $7.30 \pm 0.50$ & $27.00 \pm 5.00$ & $36,37,35,12$ \\
\hline $000418 \ldots \ldots \ldots \ldots \ldots$ & 1.1181 & 20.00 & 15,1000 & $0.997 \pm 0.018$ & $25.00 \pm 5.00$ & $27.00_{-14.00}^{+250.00}$ & $21,38,38,12$ \\
\hline 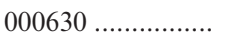 & & 2.00 & 25,100 & & $>4.0$ & $\ldots$ & 30,39 \\
\hline 000911 ................... & 1.0585 & 230.00 & 15,8000 & $0.508 \pm 0.063$ & $<1.5$ & $\ldots$ & $40,40,40$ \\
\hline 000926 ................... & 2.0369 & 6.20 & 25,100 & $3.912 \pm 1.328$ & $1.80 \pm 0.10$ & $27.00 \pm 3.00$ & $41,42,43,43$ \\
\hline $010222 \ldots \ldots \ldots \ldots \ldots \ldots$ & 1.4768 & $120.00 \pm 3.00$ & 2,700 & $1.115 \pm 0.004$ & $0.93_{-0.06}^{+0.15}$ & 1.70 & $44,45,46,12$ \\
\hline 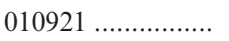 & 0.4509 & $15.40 \pm 0.20$ & 8,400 & $1.475 \pm 0.289$ & $33.00 \pm 6.50$ & $\ldots$ & $47,48,49$ \\
\hline $011121 \ldots \ldots \ldots \ldots \ldots$ & 0.3620 & 24.00 & 25,100 & $4.996 \pm 2.006$ & $>7.0$ & $\ldots$ & $50,51,51$ \\
\hline $011211 \ldots \ldots \ldots \ldots \ldots$ & 2.1400 & 5.00 & 40,700 & $1.068 \pm 0.084$ & $1.77 \pm 0.28$ & $\ldots$ & $52,53,54$ \\
\hline $020124 \ldots \ldots \ldots \ldots \ldots . .$. & $\ldots$ & 3.00 & 8,85 & $\ldots$ & $\ldots$ & $\ldots$ & 55 \\
\hline 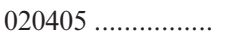 & 0.6899 & $38.00 \pm 4.00$ & 50,700 & $1.318 \pm 0.096$ & $1.67 \pm 0.52$ & $\ldots$ & 47,56 \\
\hline $020331 \ldots \ldots \ldots \ldots \ldots$ & & 0.40 & 8,40 & & . & $\ldots$ & 57 \\
\hline $020813 \ldots \ldots \ldots \ldots \ldots$ & 1.2540 & 38.00 & 25,100 & $4.336 \pm 1.682$ & $0.43 \pm 0.06$ & & $58,59,58$ \\
\hline $021004 \ldots \ldots \ldots \ldots \ldots$ & 2.3320 & 3.20 & 7,400 & $1.188 \pm 0.098$ & $7.60 \pm 0.30$ & $30.00_{-27.00}^{+270.00}$ & $60,61,62,63$ \\
\hline $021211 \ldots \ldots \ldots \ldots \ldots$ & 1.0060 & 1.00 & 8,40 & $12.345 \pm 6.462$ & $\ldots$ & $\ldots$ & 64,65 \\
\hline
\end{tabular}

Notes.- References are given in order for the redshift, fluence, jet-break time $\left(t_{\text {jet }}\right)$, and density $(n)$. Where several redshift measurements are available for the same burst, we choose the most precise determination. For the fluence measurement, we choose the measurement with the most precisely measured prompt burst spectrum. For bursts with more than one $t_{\text {jet }}$ or $n$ measurement, we choose the reference where the most broadband data were used for afterglow modeling, preferring modeling that includes radio afterglow measurements

a The energy range over which the fluence was reported.

b The prompt burst spectral $k$-correction, as defined in Bloom et al. $2001 \mathrm{~b}$, used to transform the observed fluence $S_{\gamma}$ in the particular bandpass to a standard rest-frame bandpass of 20-2000 keV.

REFERENCES.- (1) Bloom, Djorgovski, \& Kulkarni 2001a; (2) Frontera et al. 1998; (3) Bloom et al. 1998a; (4) Piran, Jimenez, \& Band 2000; (5) Frail, Waxman, \& Kulkarni 2000a; (6) Djorgovski et al. 2001; (7) Kulkarni et al. 1998a; (8) Groot et al. 1998; (9) Yost et al. 2002; (10) Galama et al. 1999; (11) Jaunsen et al. 2001b; (12) Panaitescu \& Kumar 2002; (13) Djorgovski, Bloom, \& Kulkarni 2000; (14) Woods, Kippen, \& Connaughton 1998; (15) Halpern \& Fesen 1998; (16) Djorgovski et al. 1998; (17) Frail et al. 2003; (18) Frontera et al. 2000; (19) Frail et al. 1999; (20) Kulkarni et al. 1999; (21) Bloom et al. 2003; (22) Vreeswijk et al. 2001; (23) Harrison et al. 1999; see also Stanek et al. 1999; (24) Le Floc'h et al. 2002; (25) Amati et al. 2000; (26) Masetti et al. 2000; (27) Frontera et al. 2001; (28) Fruchter et al. 2000; (29) Castro-Tirado et al. 2001; (30) Hurley et al. 2000; (31) Sagar et al. 2000; (32) Vreeswijk et al. 1999; (33) Halpern et al. 2000; (34) Andersen et al. 2000; (35) Piro et al 2002; (36) Castro et al. 2000a; (37) Berger et al. 2000; (38) Berger et al. 2001; (39) Fynbo et al. 2001; (40) Price et al. 2002a; (41) Castro et al. 2000b; (42) Price et al. 2001; (43) Harrison et al. 2001; (44) Mirabal et al. 2002; (45) in't Zand et al. 2001; (46) Galama et al. 2003b; see also Jha et al. 2001; (47) Price et al. 2002c; (48) Ricker et al. 2002c; (49) Price et al. 2003a; (50) Garnavich et al. 2003; (51) Price et al. 2002b; (52) Holland et al. 2002; (53) Frontera et al. 2001; (54) O. Fox et al. 2003, in preparation; (55) Ricker et al. 2002a; (56) Price et al. 2003c; (57) Ricker et al. 2002b; (58) Barth et al. 2003; (59) Hurley et al. 2002; (60) Matheson et al. 2003; (61) Lamb et al. 2002; (62) Pandey et al. 2002; (63) Schaefer et al. 2003; (64) Vreeswijk et al. 2002; (65) Crew et al. 2002.

principle, for measuring the geometric parameters of the universe ("cosmography").

A Hubble diagram, apparent distance modulus versus redshift, is one way to visualize the standard candle relation, now where the distance in the vertical axis corresponds to magnitude (i.e., $2.5 \mathrm{mag}$ is a difference of a factor of 10). In
Figure 2, we present the apparent GRB distance moduli for the 24 GRBs where such measurements (or constraints thereon) are now possible. The solid curves show the theoretical distance moduli in six cosmologies. For each cosmology, we recompute $\mathscr{E}_{\gamma}$ using the median of the same wellstudied bursts as described above. This value of $\mathscr{E}_{\gamma}$ is then 
TABLE 2

Computed GRB Energetics and the Apparent GRB Distance Modulus

\begin{tabular}{|c|c|c|c|c|c|c|c|c|}
\hline \multirow[b]{3}{*}{ GRB } & \multirow{3}{*}{$\begin{array}{c}E_{\text {iso }}(\gamma) \\
\left(\times 10^{50} \mathrm{ergs}\right)\end{array}$} & \multirow{3}{*}{$\begin{array}{c}\theta_{\text {jet }} \\
(\mathrm{deg})\end{array}$} & \multirow{3}{*}{$\begin{array}{c}E_{\gamma}{ }^{\mathrm{a}} \\
\left(\times 10^{50} \mathrm{ergs}\right)\end{array}$} & \multicolumn{5}{|c|}{ GRB Distance Modulus } \\
\hline & & & & \multirow[b]{2}{*}{$\mathrm{DM}^{\mathrm{b}}$} & \multicolumn{4}{|c|}{ Error Budget in $\mathrm{DM}^{\mathrm{c}}$} \\
\hline & & & & & $S_{\gamma}$ & $k$ & $n$ & $t_{\text {jet }}$ \\
\hline $970228 \ldots$. & $141.60 \pm 25.19$ & & & & & & & \\
\hline 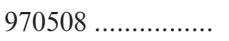 & $54.57 \pm 6.12$ & $21.63 \pm 1.67$ & $3.84 \pm 0.73$ & $45.10 \pm 0.47$ & 0.26 & 0.13 & 0.06 & 0.37 \\
\hline $970828 \ldots \ldots \ldots \ldots \ldots . . .$. & $2198.20 \pm 239.59$ & $7.13 \pm 0.50$ & $17.00 \pm 3.03$ & $43.86 \pm 0.45$ & 0.26 & 0.11 & 0.06 & 0.34 \\
\hline 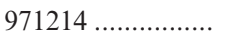 & $2105.36 \pm 257.65$ & $>5.54 \pm 0.23$ & $>9.84 \pm 1.47$ & $<47.83 \pm 0.37$ & 0.26 & 0.18 & 0.06 & 0.19 \\
\hline 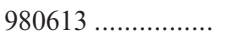 & $53.55 \pm 10.39$ & $>12.57 \pm 0.58$ & $>1.28 \pm 0.28$ & $<47.03 \pm 0.54$ & 0.38 & 0.33 & 0.06 & 0.19 \\
\hline 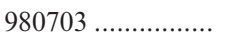 & $601.20 \pm 65.54$ & $11.21 \pm 0.81$ & $11.47 \pm 2.07$ & $44.31 \pm 0.45$ & 0.26 & 0.11 & 0.22 & 0.27 \\
\hline $990123 \ldots \ldots \ldots \ldots \ldots$ & $14378.92 \pm 1778.12$ & $4.93 \pm 0.43$ & $53.14 \pm 11.33$ & $44.00 \pm 0.53$ & 0.26 & 0.19 & 0.06 & 0.42 \\
\hline $990506 \ldots \ldots \ldots \ldots \ldots . .$. & $8614.96 \pm 1012.56$ & $\ldots$ & $\ldots$ & $\ldots$ & $\ldots$ & $\ldots$ & $\ldots$ & $\ldots$ \\
\hline 990510 ................... & $1763.49 \pm 200.18$ & $3.36 \pm 0.21$ & $3.04 \pm 0.51$ & $47.13 \pm 0.42$ & 0.26 & 0.14 & 0.28 & 0.12 \\
\hline 990705 ................. & $2559.52 \pm 203.18$ & $5.33 \pm 0.41$ & $11.05 \pm 1.91$ & $43.98 \pm 0.43$ & 0.06 & 0.20 & 0.06 & 0.37 \\
\hline 990712 ................... & $49.70 \pm 5.27$ & $>40.80 \pm 1.70$ & $>12.08 \pm 1.60$ & $<42.13 \pm 0.33$ & 0.12 & 0.25 & 0.06 & 0.18 \\
\hline 991208 ..................... & $1121.55 \pm 329.97$ & $<8.64 \pm 0.77$ & $<12.72 \pm 4.38$ & $>43.35 \pm 0.86$ & 0.26 & 0.71 & 0.36 & 0.19 \\
\hline $991216 \ldots \ldots \ldots \ldots \ldots . . .$. & $5353.69 \pm 593.92$ & $4.57 \pm 0.72$ & $17.03 \pm 5.65$ & $44.02 \pm 0.83$ & 0.26 & 0.12 & 0.46 & 0.62 \\
\hline $000131 \ldots \ldots \ldots \ldots \ldots . .$. & $11618.99 \pm 1767.35$ & $<4.68 \pm 0.21$ & $<38.66 \pm 6.79$ & $>47.06 \pm 0.44$ & 0.26 & 0.30 & 0.06 & 0.19 \\
\hline $000210 \ldots \ldots \ldots \ldots \ldots . .$. & $1693.26 \pm 140.51$ & $>6.84 \pm 0.28$ & $>12.04 \pm 1.40$ & $<43.90 \pm 0.29$ & 0.08 & 0.20 & 0.06 & 0.19 \\
\hline 000301C.. & $437.49 \pm 62.21$ & $13.14 \pm 0.51$ & $11.46 \pm 1.86$ & $46.30 \pm 0.40$ & 0.26 & 0.26 & 0.12 & 0.13 \\
\hline 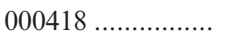 & $751.37 \pm 76.31$ & $22.30 \pm 6.34$ & $56.20 \pm 32.06$ & $42.97 \pm 1.43$ & 0.26 & 0.05 & 1.35 & 0.37 \\
\hline 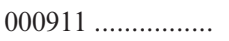 & $3954.79 \pm 630.19$ & $<5.63 \pm 0.25$ & $<19.09 \pm 3.48$ & $>44.00 \pm 0.46$ & 0.26 & 0.32 & 0.06 & 0.19 \\
\hline $000926 \ldots \ldots \ldots \ldots \ldots$ & $2797.36 \pm 990.19$ & $6.16 \pm 0.31$ & $16.17 \pm 5.95$ & $45.93 \pm 0.92$ & 0.26 & 0.87 & 0.07 & 0.10 \\
\hline $010222 \ldots \ldots \ldots \ldots \ldots$ & $8578.41 \pm 216.66$ & $3.20 \pm 0.13$ & $13.33 \pm 1.13$ & $45.28 \pm 0.21$ & 0.06 & 0.01 & 0.06 & 0.19 \\
\hline 010921 ................... & $136.11 \pm 26.70$ & $31.19 \pm 2.46$ & $19.67 \pm 4.90$ & $41.71 \pm 0.62$ & 0.03 & 0.50 & 0.06 & 0.36 \\
\hline $011121 \ldots \ldots \ldots \ldots \ldots$ & $455.59 \pm 188.54$ & $>15.35 \pm 1.00$ & $>16.26 \pm 7.05$ & $<41.35 \pm 1.08$ & 0.26 & 1.03 & 0.06 & 0.19 \\
\hline 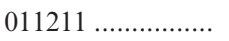 & $672.34 \pm 85.68$ & $6.38 \pm 0.40$ & $4.17 \pm 0.74$ & $47.54 \pm 0.45$ & 0.26 & 0.20 & 0.06 & 0.30 \\
\hline $020405 \ldots \ldots \ldots \ldots \ldots$ & $720.08 \pm 92.23$ & $7.81 \pm 0.93$ & $6.68 \pm 1.80$ & $43.99 \pm 0.67$ & 0.27 & 0.19 & 0.06 & 0.58 \\
\hline $020813 \ldots \ldots \ldots \ldots \ldots$ & $7749.76 \pm 3105.50$ & $3.13 \pm 0.23$ & $11.58 \pm 4.94$ & $45.00 \pm 1.07$ & 0.26 & 1.00 & 0.06 & 0.26 \\
\hline 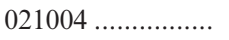 & $556.01 \pm 72.10$ & $12.67 \pm 4.51$ & $13.53 \pm 9.77$ & $46.48 \pm 1.80$ & 0.26 & 0.21 & 1.77 & 0.07 \\
\hline $021211 \ldots \ldots \ldots \ldots \ldots$ & $378.24 \pm 201.58$ & $\ldots$ & $\ldots$ & $\ldots$ & $\ldots$ & $\ldots$ & $\ldots$ & $\ldots$ \\
\hline
\end{tabular}

a $E_{\gamma}$ is computed using eq. (1). The errors on the four observable parameters $k, t_{\mathrm{jet}}, S_{\gamma}$, and $n$ are taken from Table 1 . When no errors are known for a given parameter we assume a fractional error of $10 \%$. We have taken $\Omega_{m}=0.3$ and $\Omega_{\Lambda}=0.7$ for these energetics calculations.

b DM is the apparent GRB distance modulus calculated from eq. (3), assuming $\mathscr{E} \gamma=1.33$ foe. The cosmology as noted above was used for the calculation.

c These columns give the error contribution to the DM measurement from each observable parameter. The error on DM is the quadrature sum of these four numbers. Divide these numbers by 2.5 to get fractional error contribution to $E_{\gamma}$.

used to set the zero point of the apparent GRB DM measurements from equation (3).

Aside from an overall zero point, the values of the apparent GRB DM are almost ${ }^{7}$ entirely dependent on observables, and not cosmology, yet the theoretical DM curve are sensitive to cosmology $\left(\propto 5 \log d_{l}\right)$. That the theoretical DM curves trace the apparent GRB DM measurements in Figure 2 is simply a recasting of the standard candle energy result.

\subsection{Limitations in Cosmographic Applications}

One striking feature of Figure 2 is that the data appear to be well represented by the theoretical curves in all six (rather different) cosmologies, with no apparent discrepant trend with redshift. The rms scatter about the respective $\mathscr{E}_{\gamma}$ is

\footnotetext{
7 There is a small dependence of $\theta_{\text {jet }}$ upon cosmology, such that for small opening angles, the value of DM goes as $1.25 \times \log _{10} r D_{l}(z)$, for a ratio, $r D_{l}(z)$, of luminosity distances vs. redshift relation in two different cosmologies. Even at the extrema, for most plausible cosmologies, this ratio is less than about 2 for redshifts less than 5 . Therefore, at most, the DM constructed from observable parameters could be systematically off by $\lesssim 0.35$; that is, the role of cosmology in determining the RHS of eq. (4) is usually small compared with the error on the observables themselves.
}

about the same in each cosmology $(\approx 0.35 \mathrm{dex})$, dominated by the outliers in the distribution. The main difference between various cosmological formulations is that the standard energy varies by a factor of $\sim 3$, all about energies of $\sim 1$ foe.

We explain the insensitivity of the energy data to cosmographic discrimination as follows. Without an a priori knowledge of the true standard energy release - either via observations of a local sample or from theoretical studiesthe data themselves are used to find the standard energy, setting the zero point for the DM measurements. This is akin to side-stepping the cosmological distance "ladder." Indeed, without an anchor, the divergence of theoretical DMs in different cosmologies at large redshifts is subtle and hence more difficult to observe. In other words, unlike with other cosmological tools such as with SNe Ia (with local calibrations) or the Sunyaev-Zeldovich effect (which depend only on local physics), any cosmographic discrimination with GRBs is performed differentially. As can be seen in Figure 2, the current scatter in GRB energies is simply too large for any apparent differential trend with redshift to be seen. The detection of lower redshift $(z \lesssim 0.3)$ bursts with Swift would help pin down $\mathscr{E}_{\gamma}$ and increase the sensitivity of the sample to cosmology. 


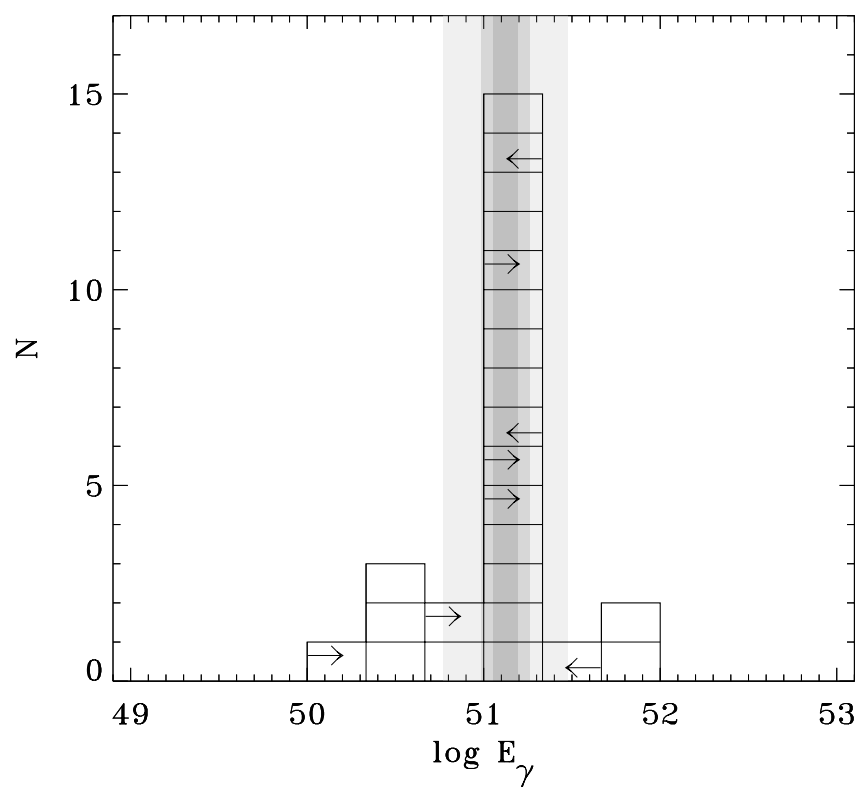

FIG. 1.-Histogram of GRB energies $\left(E_{\gamma}\right)$ with three equal logarithmic spacings per decade. The histogram shows a narrow distribution of GRB energies about the standard energy $\mathscr{E}_{\gamma}=1.33$ foe, with an error of $\sigma=0.07$ dex. The observed burst-to-burst rms spread is 0.35 dex (a factor of 2.23) about this value. Bands of 1,2 , and $5 \sigma$ about the standard energy are shown. There are five identifiable outliers, which lie more than $5 \sigma$ from the mean; however, there is currently no basis other than discrepant energy to exclude these bursts from the sample. In Fig. 3 we identify two bursts (not shown here) that are discrepant in both energy and afterglow properties. [See the electronic edition of the Journal for a color version of this figure.]

\section{TOWARD A MORE HOMOGENEOUS SET}

As noted in $\S 1$, cosmographic applications benefit enormously if the objects are well understood physically. Lacking a clear understanding (as is arguably still the case with $\mathrm{SNe}$ Ia), an astronomical appreciation of the diversity of the phenomenon is critical. In short, if the scatter on $E_{\gamma}$ is ever to be reduced a priori, it is essential that we develop the ability to identify subclasses from observations so that a homogeneous set of GRBs can be identified for cosmographic applications.

We already know of at least one member of a distinct subclass of GRBs: the GRB associated with SN 1998bw (Galama et al. 1998; Kulkarni et al. 1998b). Indeed, GRB 980425 , with an energy release of $E_{\gamma} \lesssim 7 \times 10^{47}$ ergs, is clearly abnormal (see Fig. 3). Bloom et al. (1998b) suggested that such low-luminosity GRBs that are associated with supernovae to be "S-GRBs." Recently, Norris (2002) identified $\sim 90$ such low-redshift S-GRB candidates in the $B A T S E$ sample. Without appealing to gamma-ray properties, such GRBs are readily identified by their low redshift and, possibly, the dominance of the associated supernova emission over the afterglow light.

Of the more classical "cosmological GRBs," those with known redshifts beyond $\gtrsim 100 \mathrm{Mpc}$ or associated with distant galaxies, we can use Figure 3 help identify classes of outliers. In Figure 3, we show the $E_{\gamma}$ distribution energy versus redshift for the 24 bursts with known redshift. We also show the trajectory of $E_{\gamma}$ for the five bursts where no redshift is known. These curves were found by computing the individual $k$-corrections and $f_{b}$ as a function of redshift.

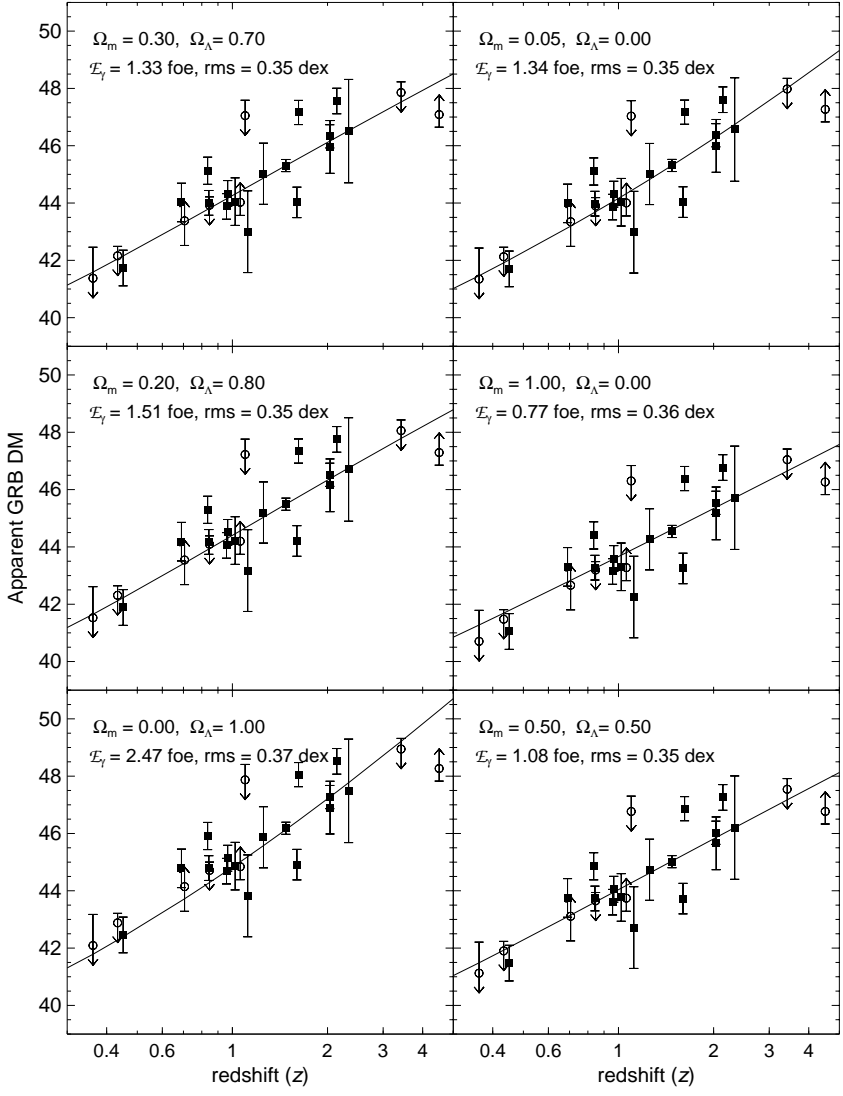

FIG. 2.-GRB Hubble diagram illustrating the difficulty in making cosmographic measurements with GRBs. Shown are Hubble diagrams for six different cosmologies for the 24 GRBs with known redshift and a measurement or constraint on $t_{\text {jet }}$. The solid squares represent the apparent GRB distance modulus in the given cosmology with associated $1 \sigma$ errors. Open circles show those sources with upper or lower limits on the DM measurement. The solid curves are the theoretical DM for the given cosmology. Although the values of $\mathscr{E}_{\gamma}$ vary by more than a factor of 3 over the cosmologies shown, the observed variance about the theoretical curves is almost the same $(\approx 0.35 \mathrm{dex})$; we discuss in the text why this is so. Clearly, the current sample cannot distinguish between a wide range of cosmologies. Here and throughout the paper, we use $H_{0}=65 \mathrm{~km} \mathrm{~s}^{-1} \mathrm{Mpc}^{-1}$.

Note that the implied $E_{\gamma}$ curve begins to flatten at high redshift as a result of the mitigation between larger $E_{\text {iso }}(\gamma)$ and smaller inferred $f_{b}$. From Figure 3, it can be seen that the energy release in GRB 980329 and GRB 000630 could have been consistent with $\mathscr{E}_{\gamma}$ for redshift $z \gtrsim 1.5$. The present observations provide only a weak lower limit to the energy of GRB 981226.

\section{1. "Fast-fading GRBs"-A New Subclassification?}

As is readily seen in Figure 3, GRB 980519 and GRB 980326 stand out for being significantly lower in energy release than the cosmological set. Even at a redshift of 5, these bursts released less than 0.2 foe. Interestingly, independent of the inferred low energy, GRB 980519 and GRB 980326 have previously been recognized as peculiar in that both afterglows exhibited an unusually steep decline, flux proportional to $t^{-\alpha}$ with $\alpha \sim 2$ (instead of the usual $\alpha \sim 1$ ) at early times. We propose that bursts with early-time steeply declining afterglows may be intrinsically underenergetic and suggest a nomenclature, fast-fading GRBs 


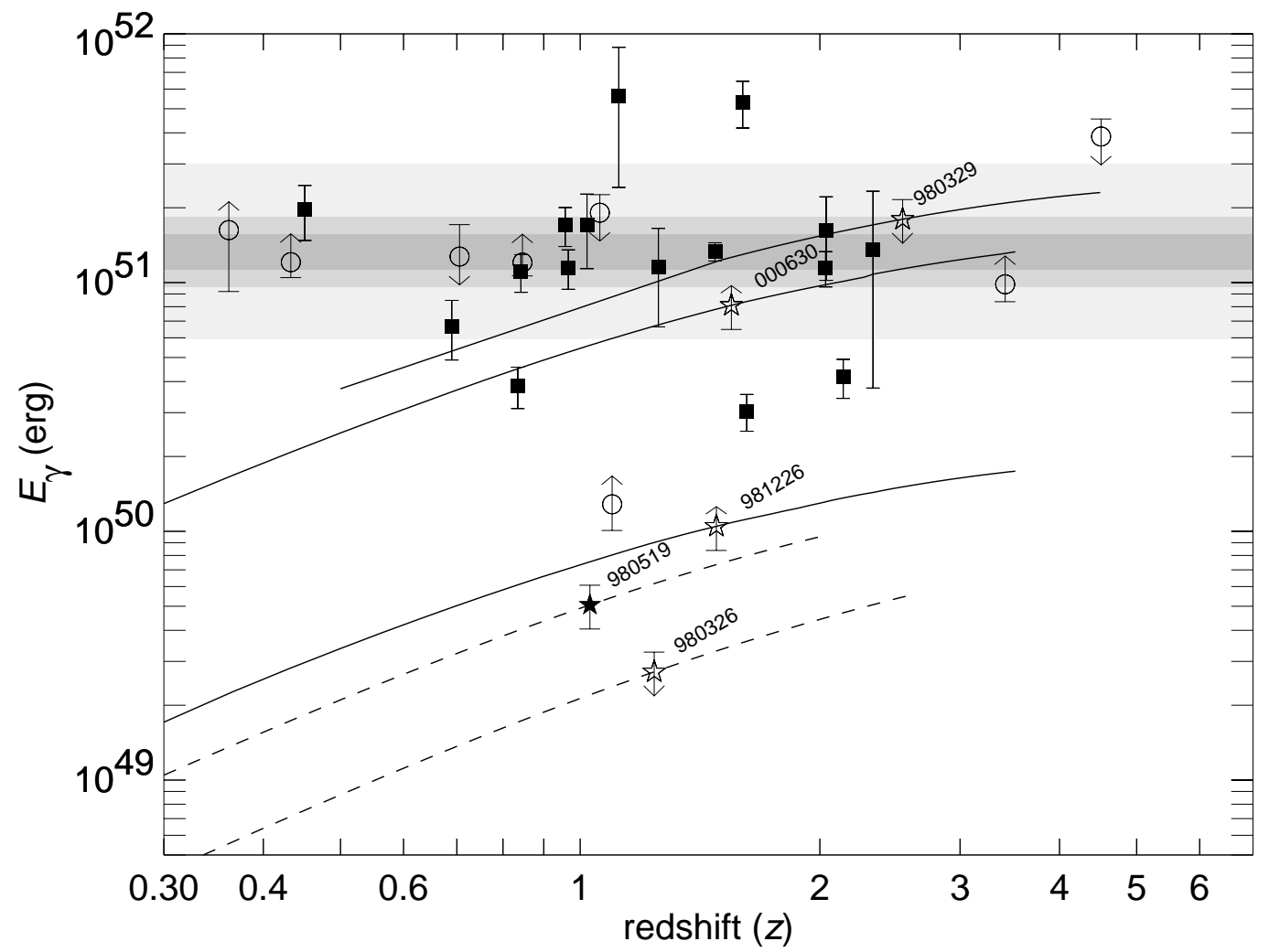

FIG. 3.-GRB energy release vs. redshift assuming a top-hat jet illustrating the diversity in GRB energies. Bands of 1, 2, and $5 \sigma$ about the mean energy $\mathscr{E}_{\gamma}=1.33$ foe are shown. Solid box and open circle symbols are the same as in Fig. 2. Plotted are the trajectories of five GRBs with no known spectroscopic redshift (stars). Redshift limits are placed from photometric observations of the afterglow (980519: Jaunsen et al. 2001a; 980326: Bloom et al. 1999; 000630: Fynbo et al. 2001; 991216: Halpern et al. 2000) assuming no extinction from the redshifted Ly $\alpha$ forest in the most blue filter. The photometric redshift of the host galaxy of 980329 is from Jaunsen et al. (2003). While the energies of GRB 980329 and GRB 000630 could be consistent with the standard value at redshifts beyond $z \sim 1.5$, at no redshift could the energies of GR B 980326 and GRB 980519 (dashed lines) be consistent unless the densities were significantly higher than the canonical value of $10 \mathrm{~cm}^{-3}$. Interestingly, these two bursts are associated with having occurred in a wind-blown density environment. While most longduration GRBs appear to fall within a narrow range of energies, this shows there are several exceptional outliers, with demonstrably different afterglow properties from other bursts; we refer to such bursts as fast-fading GRBs or f-GRBs. [See the electronic edition of the Journal for a color version of this figure.]

or "f-GRBs" (in analogy with the "Branch peculiars" of SNe Ia), for such GRBs.

While the identification of f-GRBs, based upon fastdeclining afterglows, is also an appeal to the physical state of those systems, under the assumption of an interstellar medium (ISM) density, we cannot identify any unifying observation of the five closer energy outliers (970508, 990123, 990510, 000418, and 011211) that belie some unifying physical process. We therefore cannot (yet) distinguish such bursts a priori from the remainder of the set.

An f-GRB could be either a genuinely low-energy event or a typical-energy burst that occurs in a particularly dense medium (e.g., Dai \& Lu 2001); note that for the best-studied f-GRB - 030329 (see below) - there is no indication of a dense medium. In formulating our energy measurements, we have explicitly assumed that the afterglow emission before the jet break arises in a medium where density is constant and homogeneous [i.e., $\rho(r) \propto r^{0}$ ]. Since the environments of a least some GRBs are thought to be clumpy (Lazzati et al. 2002; Bersier et al. 2003; Heyl \& Perna 2003) and wind-blown [i.e., $\rho(r) \propto r^{-2}$; Chevalier \& Li 1999; Price et al. 2002b], this is clearly not the case for all GRBs in our sample; therefore, our estimations of $f_{b}$ may be inappropriate for some GRBs. As in the homogeneous case, the dynamics of $\Gamma$ in jetted GRB within winds should be insensitive to the jet edge so long as $\Gamma \lesssim 1 / \theta_{\text {jet. }}$. We can therefore cal- culate $\theta_{\text {jet }}$ (wind) by inverting the equation for $\Gamma$ in equation (5) of Chevalier \& Li (2000):

$$
\begin{aligned}
\theta_{\text {jet }}(\text { wind })= & 0.169\left(\frac{1+z}{2}\right)^{-1 / 4}\left[\frac{E_{\text {iso }}(\gamma)}{10^{52} \mathrm{ergs}}\right]^{-1 / 4} \\
& \times A *^{1 / 4}\left(\frac{t_{\text {jet }}}{\text { day }}\right)^{1 / 4} .
\end{aligned}
$$

For all bursts in our sample for which $A *$ has been reported, we calculate $\theta_{\text {jet }}$ (wind) following equations (1) and (6). The results are as follows $\left[\theta_{\text {jet }}(\right.$ wind $), E_{\gamma} / 10^{50} \mathrm{ergs} ; A *$ reference]: 970508 (20.81, 3.560; Panaitescu \& Kumar 2002); 980519 (4.88, 6.262; Panaitescu \& Kumar 2002) (5.13, 6.941; Chevalier \& Li 1999); 991208 (<5.29, <4.771; Li \& Chevalier 2001); 990123 (2.82, 17.390; Panaitescu \& Kumar 2002); 000301C (8.15, 4.411; Li \& Chevalier 2001); 000418 (11.78, 15.834; Panaitescu \& Kumar 2002); 000926 (5.24, 11.689; Panaitescu \& Kumar 2002); 010222 (1.93, 4.891; Panaitescu \& Kumar 2002); 011121 (>4.47, >1.390; Price et al. 2002b). (We assume $z=2.5$ for GRB 980519. Note that the wind hypothesis for GRB 991208 is strongly disfavored with late-time radio modeling of Galama et al. 2003a.) Comparison with Table 2 shows that the inferred values for $\theta_{\text {jet }}$ and $E_{\gamma}$ are usually smaller under the wind assumption. Uncertainties in $A *$ are not generally provided, 
so it is unclear if the energy determinations are consistent between winds and ISM models. GRB 970508 is underluminous by a factor of $\sim 3$ in both the ISM and wind cases. Both GRB 000418 and GRB 990123, overluminous in the ISM case, are more consistent with the standard $\mathscr{E}_{\gamma}$ in the wind case.

Only GRB 980519 and GRB 011121 show convincing evidence for a wind-stratified medium under the assumption of a top-hat jet model (GRB 980326 is consistent with a wind; Bloom et al. 1999). As seen, the energy release in GRB 011121 is similar in both the wind and ISM cases. Interestingly, the energy release in GRB 980519, again assuming $z=2.5$ and a wind-blown circumburst medium, is $6.3 \times 10^{50} \mathrm{ergs}$ and thus consistent with $\mathscr{E}_{\gamma}$. Therefore, it may be reasonable to suspect that all $\mathrm{f}-\mathrm{GRB}$ are bursts that occur in wind-stratified media (but not necessarily the converse).

\section{DISCUSSION AND CONCLUSIONS}

With nine new bursts suitable for $E_{\gamma}$ measurement after FKS01, the evidence for a standard release of energy in GRBs persists. By incorporating more realistic measurements of ambient density and under the assumption of a top-hat jet and constant ambient density media, we find a standard candle energy release of $\mathscr{E}_{\gamma}=1.33$ foe, with a burst-to-burst scatter of 0.35 dex about this value. Although this result makes it interesting to consider cosmographic applications of GRBs, we have shown that without a local calibration of the true energy release, the observed scatter is simply too large to make any meaningful impact on cosmographic measurements.

The most obvious way to reduce the scatter on $\mathscr{E}_{\gamma}$ is to simply remove outliers from the sample, based purely upon discrepant energy measurements. However, this is a dangerously circular approach since it is particularly these apparent outliers in energy, at high redshift especially, that place the most meaningful constraints for cosmography.

Still, at this stage, we are confined to first recognize heterogeneity in the context of a cosmological model and then find similarities between discrepant bursts, based upon observations or theory that are complementary to energy. To this end, we have noted that both GRB 980519 and GRB 980326 are low-energy bursts and stand out based upon afterglow observations.

Indeed, lacking firm theoretical predictions for subclassification, we have proposed that there are at least two subclasses-S-GRBs and f-GRBs-of GRBs that can be typed or classified from simple observations. Both groups were first identified by low inferred gamma-ray energy releases. Now, S-GRBs are characterized by low redshift ${ }^{8}$ and $\mathrm{f}-\mathrm{GRBs}$ are identified by the rapid fading, $t^{-2}$, of their afterglow at early times, $t \lesssim 0.5$ days (rest frame).

The advantage of empirical distance indicators (e.g., Schaefer 2003) is that they can be entirely based on the gamma-ray observations and do not require (once calibrated) additional time-consuming observations from the ground. However, as with many empirical indicators, the

\footnotetext{
${ }^{8}$ The origin of gamma-ray emission in S-GRBs has now been discussed in some detail (e.g., Tan, Matzner, \& McKee 2001), and it is reasonable to infer that the gamma-ray emission arises from entirely different process as compared to the internal shocks of cosmologically located GRBs.
}

method relies on poorly understood physical mechanisms (however, see Salmonson 2000). The sheer bewildering diversity in any high-energy property of GRBs-richness of profiles, overall profile shapes, fluences, peak luminosities, and even peak energies - should give pause to claims of the predictive power of distance indicators in the absence of a realistic physical model for the emission mechanisms.

The physical uncertainty aside, it is clear that the highenergy properties can and have provided gross distinctions. The well-known separation in energy and spectra between short bursts and hard bursts (Kouveliotou et al. 1993), for example, has led to the belief that bursts from each class may originate from different progenitors. As a more recent example, we note that Norris (2002) has used the lagluminosity relation to identify a subsample of long-duration bursts whose properties distinguish them from the general population. However, we question whether empirical relations have demonstrated that they possess the precision necessary to advance cosmography. There is little reason based on the limited understanding of the GRB phenomenon to strongly justify an optimistic expectation in this direction.

The approach we have used in FKS01 and herein has the advantage that it is based upon energy release, a fundamental quantity in explosions; on physical grounds, it is reasonable to expect that such a quantity could be standard (or at least amenable to calibration) despite the rich diversity in GRB properties. The standard energy result is corroborated by other studies (which do not rely on the gamma-ray data), namely, multiwavelength afterglow modeling and X-ray observations (Panaitescu \& Kumar 2001; Piran et al. 2001; Berger et al. 2003).

We stress that if GRBs are ever to be used for cosmographic purposes, there must be a significantly increased understanding in the diversity of the phenomenon (let alone the secular evolution with redshift). To this end, using simple observations, at least two subclasses of GRBs, the S-GRBs and the f-GRBs, may be readily recognized. For the short term, we believe that the identification of such bursts will provide informative direction to hone in on GRB progenitors. To weed out such bursts and create a more homogeneous sample for cosmography, however, our proposed identification methods have the disadvantage of requiring significant follow up observations. Nevertheless, it is possible that in the SWIFT era, dedicated facilities will undertake the necessary follow-up observations quite routinely.

After this paper was submitted, GRB 030329 was discovered and found to fade rapidly at early times $\left(F_{\nu} \propto t^{1.97 \pm 0.12}\right.$; Price et al. 2003b), suggesting that the burst fit our definition of an f-GRB. Using the same formalism above, we find $E_{\gamma}=(8.18 \pm 1.00) \times 10^{49}$ ergs assuming a constant density ISM and $n=10 \mathrm{~cm}^{-3}$ or $1.3 \times 10^{50}$ ergs assuming a wind with $A *=1.0$. In the former case, GRB 030329 is underluminous by a factor of 16.3 from the $\mathscr{E}_{\gamma}$ found herein. Thus, GRB 030329 joins GRB 980519 and GRB 980326 in the same subclass on both energetics and phenomenological grounds.

Given the connection of GRB 030329 with a supernova (Stanek et al. 2003), we propose that all f-GRBs may be associated with supernovae. Obviously with afterglows that fade fast there is an increasing likelihood of detecting a supernova component (the nondetection of a SN component in the optical light curve of GRB 980519 [see Jaunsen 
et al. 2001a] may simply be due to $z \gtrsim 1$ of this burst). However, we suggest that the connection may be more than due to observational bias; instead, long-duration GRBs could arise from two populations of bursts (Chevalier \& Li 1999), with $\mathrm{f}$-GRBs from massive stars that produce winds prior to the GRB and a concurrent supernova with the GRB event. Alternatively, especially in light of the detection of a supernova component with GRB 011121 (Bloom et al. 2002; Garnavich et al. 2003), we favor the hypothesis that all long-duration GRBs also lead to supernovae, with the diversity in GRB energies set by a progenitor-dependent ratio of the energy channeled into the relativistic jets to the energy powering the explosion the massive star progenitor.
Many thanks to the referee for illuminating comments. J. S. B. gratefully acknowledges a fellowship from the Harvard Society of Fellows and the generous research support from the Harvard-Smithsonian Center for Astrophysics. J. S. B. also thanks J. M. Paredes and the Astronomy department at the Universitat de Barcelona for their hospitality and funding support. S. R. K. acknowledges support from NASA and the NSF. We thank A. Friedman and R. Perna for helpful discussions. The National Radio Astronomy Observatory is a facility of the National Science Foundation operated under cooperative agreement by Associated Universities, Inc.
Amati, L., et al. 2000, Science, 290, 953

Andersen, M. I., et al. 2000, A\&A, 364, L54

Barth, A. J., et al. 2003, ApJ, 584, L47

Berger, E., Kulkarni, S. R., \& Frail, D. A. 2003, ApJ, 590, 379

Berger, E., et al. 2000, ApJ, 545, 56 2001, ApJ, 556, 556

Bersier, D., et al. 2003, ApJ, 584, L43

Blandford, R. D., \& McKee, C. F. 1976, Phys. Fluids B, 19, 1130

Bloom, J. S., Berger, E., Kulkarni, S. R., Djorgovski, S. G., \& Frail, D. A 2003, AJ, 125, 999

Bloom, J. S., Djorgovski, S. G., \& Kulkarni, S. R. 2001a, ApJ, 554, 678

Bloom, J. S., Djorgovski, S. G., Kulkarni, S. R., \& Frail, D. A. 1998a, ApJ, $507, \mathrm{~L} 25$

Bloom, J. S., Frail, D. A \& Sari, R. 2001b, AJ, 121, 2879

Bloom, J. S., Kulkarni, S. R., Harrison, F., Prince, T., Phinney, E. S., \&

Frail, D. A. 1998b, ApJ, 506, L105

Bloom, J. S , et al 1999, Nature, 401, 453 2002, ApJ, 572, L45

Branch, D., Fisher, A., \& Nugent, P. 1993, AJ, 106, 2383

Castro, S. M., Diercks, A., Djorgovski, S. G., Kulkarni, S. R., Galama, T. J., Bloom, J. S., Harrison, F. A., \& Frail, D. A. 2000a, GCN Circ. 605 (http://gcn.gsfc.nasa.gov/gcn/gcn3/605.gen3)

Castro, M., Djorgovski, S. G., Kulkarni, S. R., Bloom, J. S., Galama, T. J., Harrison, F. A., \& Frail, D. A. et al. 2000b, GCN Circ. 851 (http://gcn.gsfc.nasa.gov/gen/gcn3/851.gcn3)

Castro-Tirado, A. J., et al. 2001, A\&A, 370, 398

Chevalier, R. A., \& Li, Z.-Y. 1999, ApJ, 520, L29

$2000, \mathrm{ApJ}, 536,195$

Crew et al. 2002, GCN Circ. 1734 (http://gcn.gsfc.nasa.gov/gcn/gcn3/ 1734.gcn3)

Dai, Z. G., \& Lu, T. 2001, A\&A, 367, 501

Diorgovski, S. G., Bloom, J. S., \& Kulkarni, S. R. 2000, ApJ, submitted (astro-ph/0008029)

Djorgovski, S. G., Frail, D. A., Kulkarni, S. R., Bloom, J. S., Odewahn, S. C., \& Diercks, A. 2001, ApJ, 562, 654

Djorgovski, S. G., Kulkarni, S. R., Bloom, J. S., Goodrich, R., Frail, D. A., Piro, L., \& Palazzi, E. 1998, ApJ, 508, L17

Fenimore, E. E., \& Ramirez-Ruiz, E. 2000, ApJ, submitted (astro-ph/ 0004176)

Frail, D. A., Waxman, E. \& Kulkarni, S. R. 2000a, ApJ, 537, 191

Frail, D. A., et al. 1999, ApJ, 525, L81 $2000 \mathrm{~b}, \mathrm{ApJ}, 534,559$

2001, ApJ, 562, L55(FKS01)

2003, ApJ 590, 992

Frontera, F., et al. 1998, in 4th Huntsville Symp. on Gamma-Ray Bursts, ed. C. A. Meegan, R. D. Preece, \& T. M. Koshut (New York: AIP), 446 2000, ApJ, 540, 697

2001, ApJ, 550, L47

2001, GCN Circ. 1215 (http://gcn.gsfc.nasa.gov/gcn/gcn3/ 1215. $\mathrm{gcn} 3$ )

Fruchter, A., Vreeswijk, P., Hook, R., \& Pian, E. 2000, GCN Circ. 752 (http://gcn.gsfc.nasa.gov/gen/gcn3/752.gcn3)

Fynbo, J. P. U., et al. 2001, A\&A, 369, 373

Galama, T. J., Frail, D. A., Sari, R., Berger, E., Taylor, G. B., \& Kulkarni,

S. R. 2003a, ApJ, 585, 899

Galama, T. J., et al. 1998, Nature, 395, 670 1999, A\&AS, 138, 465 2003b, ApJ, 587, 135

Garnavich, P. M., et al. 2003, ApJ, 582, 924

Granot, J. \& Sari, R. 2002, ApJ, 568, 820

Groot, P. J., et al. 1998, ApJ, 502, L123

Halpern, J. P., \& Fesen, R. 1998, GCN Circ. 134

(http://gcn.gsfc.nasa.gov/gen/gcn3/134.gcn3)

Halpern, J. P., et al. 2000, ApJ, 543, 697

Harrison, F. A., et al. 1999, ApJ, 523, L121

2001, ApJ, 559, 123

\section{EFERENCES}

Heyl, J. S., \& Perna, R. 2003, ApJ, 586, L13

Holland, S. T., et al. 2002, AJ, 124, 639

Hurley, K., et al. 2000, ApJ, 534, L23

$1483 \cdot \operatorname{gcn} 3)$

in't Zand, J. J. M., et al. 2001, ApJ, 559, 710

Jaunsen, A. O., et al. 2001a, ApJ, 546, 127

.2001b, ApJ, 546, 127

2003, A\&A, 402, 125

Jha, S., et al. 2001, ApJ, 554, L155

Kouveliotou, C., Meegan, C. A., Fishman, G. J., Bhat, N. P., Briggs, M. S.,

Koshut, T. M., Paciesas, W. S., \& Pendleton, G. N. 1993, ApJ, 413, L101

Kulkarni, S. R., et al. 1998a, Nature, 393, 35 1998b, Nature, 395, 663 1999, Nature, 398, 389

Kumar, P., \& Granot, J. 2003, ApJ, 591, 1075

Lamb, D., et al. 2002, GCN Circ. 1600 (http://gcn.gsfc.nasa.gov/gcn/ $\mathrm{gcn} 3 / 1600 . \mathrm{gcn} 3)$

Lazzati, D., Rossi, E., Covino, S., Ghisellini, G., \& Malesani, D. 2002, A\&A, 396, L5

Le Floc'h, E., et al. 2002, ApJ, 581, L81

Li, W., Filippenko, A. V., Treffers, R. R., Riess, A. G., Hu, J., \& Qiu, Y. 2001, ApJ, 546, 734

Li, Z., \& Chevalier, R. A. 2001, ApJ, 551, 940

Loeb, A., \& Barkana, R. 2001, ARA\&A, 39, 19

Masetti, N., et al. 2000, A\&A, 354, 473

Matheson, T., et al. 2003, ApJ, 582, L5

Mészáros, P., Rees, M. J., \& Wijers, R. A. M. J. 1998, ApJ, 499, 301

Metzger, M. R., Djorgovski, S. G., Kulkarni, S. R., Steidel, C. C., Adelberger, K. L., Frail, D. A., Costa, E., \& Fronterra, F. 1997, Nature, 387,879

Mirabal, N., et al. 2002, ApJ, 578, 818

Norris, J. P. 2002, ApJ, 579, 386

Norris, J. P., Marani, G. F., \& Bonnell, J. T. 2000, ApJ, 534, 248

Panaitescu, A., \& Kumar, P. 2001, ApJ, 560, L49 2002, ApJ, 571, 779 2003 ApJ in press

Panaitescu, A., Mészáros, P., \& Rees, M. J. 1998, ApJ, 503, 314

Pandey, S. B., et al. 2002, Bull Astron. Soc. India, submitted (astro-ph/ 0211108)

Piran, T., Jimenez, R., \& Band, D. 2000, in 5th Huntsville Symp. on Gamma-Ray Bursts, ed. R. S. M. R. M. Kippen \& G. J. Fishman (New York: AIP), 87

Piran, T., Kumar, P., Panaitescu, A., \& Piro, L. 2001, ApJ, 560, L167

Piro, L., et al. 2002, ApJ, 577, 680

Price, P. A., et al. 2001, ApJ, 549, L7

.2002a, ApJ, 573, 85

2002b, ApJ, 572, L51

$2002 \mathrm{c}$, ApJ, 571, L12

2003a, ApJ, 584, 931

2003b, Nature, 423,844

2003c, ApJ, 589,838

Reichart, D. E., Lamb, D. Q., Fenimore, E. E., Ramirez-Ruiz, E., Cline, T. L., \& Hurley, K. 2001, ApJ, 552, 57

Rhoads, J. E. 1997a, IAU Circ. 6793, 1 1997b, ApJ, 487, L1

Ricker et al. 2002a, GCN Circ. 1220 (http://gcn.gsfc.nasa.gov/gcn/gcn3/ $1220 . \operatorname{gcn} 3$ )

2002b, GCN Circ. 1315 (http://gcn.gsfc.nasa.gov/gcn/gcn3/ 1315. $\operatorname{gcn} 3)$

.2002c, ApJ, 571, L127

Rossi, E., Lazzati, D., \& Rees, M. J. 2002, MNRAS, 332, 945

Sagar, R., Mohan, V., Pandey, A. K., Pandey, S. B., \& Castro-Tirado, A. J. 2000, Bull. Astron. Soc. India, 28, 15

Salmonson, J. D. 2000, ApJ, 544, L115

Sari, R., Piran, T., \& Halpern, J. P. 1999, ApJ, 519, L17 
Sari, R., Piran, T., \& Narayan, R. 1998, ApJ, 497, L17

Schaefer, B. 2003, ApJ, 583, L67

Schaefer, B. E., et al. 2003, ApJ, 588, 387

Spergel, D. N., et al. 2003, ApJ, submitted (astro-ph/0302209)

Stanek, K. Z., Garnavich, P. M., Kaluzny, J., Pych, W., \& Thompson, I.
1999, ApJ, 522, L39

Stanek, K. Z., et al. 2003, ApJ, 591, L17

Tan, J. C., Matzner, C. D., \& McKee, C. F. 2001, ApJ, 551, 946

van den Bergh, S., \& Pazder, J. 1992, ApJ, 390, 34
Vreeswijk, P. M., et al. 1999, GCN Circ. 496 (http://gcn.gsfc.nasa.gov/ $\mathrm{gcn} / \mathrm{gcn} 3 / 496 . \mathrm{gcn} 3)$

2001, ApJ, 546, 672

2002, GCN Circ. 1785 (http://gcn.gsfc.nasa.gov/gcn/gcn3/ 1785.gcn3)

Woods, P., Kippen, R. M., \& Connaughton, V. 1998, GCN Circ. 112 (http://gcn.gsfc.nasa.gov/gcn/gcn3/112.gcn3)

Yost, S. A., et al. 2002, ApJ, 577, 155

Zhang, B., \& Mészáros, P. 2002, ApJ, 571, 876 